\title{
Identifying dysregulated pathways in postmenopausal osteoporosis through investigation of crosstalk between pathways
}

\author{
JIAN-HUA HAN, XIAO-JUN CAI, HOU-JIE SUN, GE-HUI DONG, BIN HE, \\ HAN-XIANG ZHANG, XIN ZHOU and JIA-QIANG YAN \\ Department of Orthopedics, Zunyi First People's Hospital, Zunyi, Guizhou 563002, P.R. China
}

Received February 6, 2017; Accepted September 9, 2017

DOI: $10.3892 / \mathrm{mmr} .2017 .7703$

\begin{abstract}
The present study aimed to identify potential dysregulated pathways to further reveal the molecular mechanisms of postmenopausal osteoporosis (PMOP) based on pathway-interaction network (PIN) analysis, which considers crosstalk between pathways. Protein-protein interaction (PPI) data and pathway information were derived from STRING and Reactome Pathway databases, respectively. According to the gene expression profiles, pathway data and PPI information, a PIN was constructed with each node representing a biological pathway. Principal component analysis was used to compute the pathway activity for each pathway, and the seed pathway was selected. Subsequently, dysregulated pathways were extracted from the PIN based on the seed pathway and the increased classification accuracy, which was measured using the area under the curve (AUC) index according to 5-fold cross validation. A PIN comprising 2,725 interactions was constructed, which was used to detect dysregulated pathways. Notably, the 'mitotic prometaphase' pathway was selected and defined as a seed pathway. Starting with the seed pathway, network-based analysis successfully identified one pathway set for PMOP comprising eight dysregulated pathways (such as mitotic prometaphase, resolution of sister chromatid cohesion, mRNA splicing and mRNA splicing-major) with an AUC score of 0.85 , which may provide potential biomarkers for targeted therapy for PMOP.
\end{abstract}

\section{Introduction}

Osteoporosis (OP) is a common metabolic skeletal disease that is characterized by microarchitectural deterioration of bone tissues and reduced bone mineral density (1). An imbalance in the regulation of bone remodeling results in increased

Correspondence to: Professor Xiao-Jun Cai, Department of Orthopedics, Zunyi First People's Hospital, 98 Fenghuang Road, Huichuan, Zunyi, Guizhou 563002, P.R. China

E-mail: caijunfirst@yeah.net

Key words: postmenopausal osteoporosis, pathway interaction network, crosstalk bone fragility and enhanced fracture risk (2). Postmenopausal bone loss is a major determining factor of $\mathrm{OP}$ and is a worldwide health problem. In addition, advanced age, sex and immobilization are also main risk factors for developing OP (3). Postmenopausal OP (PMOP) is one of the two types of $\mathrm{OP}$, and may develop as a direct result from the reduced endogenous estrogen levels in menopausal women $(4,5)$. Worldwide, PMOP affects thousands of women $>50$ years old and costs healthcare systems large sums of money, which may lead to an increase in economic and social burdens $(6,7)$. Therefore, studies on PMOP-related mechanisms are urgently needed and relevant.

Numerous factors serve important roles in the etiology of PMOP. In addition to calcium, estrogen and environmental factors, previous studies have reported that there are strong genetic effects on the pathogenesis of OP in postmenopausal women (8-10). For example, certain polymorphisms in a set of genes have been revealed to be associated with Chinese women with PMOP, such as estrogen receptor 2 (11), osteoprotegerin (OPG) (12). In addition, several pathways have been identified to be related with PMOP development. Wnt/ $\beta$-catenin pathway serves an important role in PMOP by changing the ratio of receptor activator of nuclear factor- $\kappa \mathrm{B}(\mathrm{NF}-\kappa \mathrm{B})$ ligand/OPG and altering bone turnover (13). One study demonstrated the therapeutic potential of resveratrol treatment against PMOP through osteoblast differentiation via sirtuin $1 / \mathrm{NF}-\kappa \mathrm{B}$ signaling (14). However, the molecular mechanisms underlying PMOP remain unclear.

Gene expression profiles in DNA microarrays have provided key biosignatures of PMOP. Therefore, an increasing number of investigators use bioinformatics approaches to study the microarray profiles of PMOP to explore molecular mechanism underlying PMOP. A microarray profile of PMOP (E-MEXP-1618) was deposited by Reppe et al (15), who detected a set of genes that were highly associated with bone mineral density in relation to PMOP. A 2015 study that used this same data identified 482 differentially expressed genes (DEGs) that exhibited a close relationship with PMOP, such as SMAD family member 4, calcium channel voltage-dependent $\gamma 1$ and tripartite motif containing 63 (16). Many of the previous studies related to the genetics of PMOP have focused on a single gene or a single pathway; however, different pathways have crosstalk with each other, and the deregulation of one pathway may affect the activity of another. Understanding 
the interactions among and between pathways may provide information for the further exploration of the pathogenesis of PMOP.

Therefore, the present study aimed to explore the pathogenesis of PMOP using the microarray data of PMOP to detect significant pathways, with consideration of the functional dependency among pathways. These results may offer theoretical guidance for future experiments and may aid in our understanding of the pathogenesis of PMOP.

\section{Materials and methods}

Brief outline of the proposed method. Based on gene expression data, cellular pathways and protein-protein interactions (PPIs) information, the identification of dysregulated pathways using the pathway-interaction network (PIN) included three steps. In the first step, the microarray genes were mapped to the pathways and the principal component analysis (PCA) method (17) was used to compute the pathway activity for each pathway according to the sum of the expression values of all genes of this given pathway, and seed pathway was selected based on the pathway activities (the pathway of activity score with the maximum change between PMOP and normal groups was regarded as the seed pathway). In the second step, a PIN was constructed that relied on gene expression data, PPIs and cellular pathways, in which each node represented a cellular pathway. In the third step, dysregulated pathways were extracted from the PIN based on the seed pathway and increased classification accuracy, which was measured using area under the curve (AUC) indexing based on five-fold cross validation.

Data availability. Raw gene expression data were downloaded from the ArrayExpress database offered by European Bioinformatics Institute (http://www.ebi.ac.uk/arrayexpress; accession no. E-MEXP-1618) (15), which was based on the A-AFFY-44 Affymetrix GeneChip Human Genome U133 Plus 2.0 (HG-U133_Plus_2) platform. This data set included 84 iliac bone biopsy samples, comprising 45 patients with OP and 39 healthy patients, which were obtained from postmenopausal women. The inclusion criteria were an average spine T-score $>-1$ for the normal group, and an average spine T-score $<-1$ for the PMOP group.

Affymetrix CEL files and probe annotation files were downloaded, and the gene expression profile of E-MEXP-1618 was pre-treated using the Affy package of Bioconductor (18). Briefly, primary data in the CEL format was converted into expression measures, followed by background correction using robust multiarray average (19). Next, quartile data normalization was conducted through quantiles (20), and median polish was used to summarize the expression measures. Finally, a gene expression matrix that included 20,545 genes was obtained following the probes were mapped to gene symbols. Subsequently, expression scores of all genes were standardized.

Biological pathways and PPI data. A total of 1,675 predefined biological pathways were obtained from the REACTOME database (http://www.reactome.org), which is a manually curated open-source database of cellular pathways (21). Pathways comprising too few genes may not have sufficient biological information, whereas pathways having too many genes may be too generic (22). Therefore, the present study extracted a set of pathways by excluding the pathways with $<5$ genes or $>100$ genes. Following removal of these pathways, 1,189 background pathways were identified for further analysis. PPIs were downloaded from the STRING database (version 9.1; http://string-db. org) (23). The original PPI data set included 787,896 interactions among 16,730 genes. The STRING database uses confidence scores to measure how likely an association will appear. As a result, with the goal of minimizing the ambiguity, only the interactions with confidence score $>0.2$ were selected to construct the original PPI network. Taking the intersection of the original PPI network and the microarray data, a background PPI data set containing 14,917 proteins and 449,833 PPIs was identified and used for further analysis.

PIN construction. Using gene expression profiles, PPI information and pathway data, a PIN was constructed, in which each node represented a pathway and an edge was laid between two pathways when they met one of two conditions; if not, then the edges were discarded. The first condition was that these two pathways had to share at least one gene, and at least one of the common genes between two pathways had to be a DEG between the PMOP and normal groups. In the present study, DEGs were identified using Student's t-test with a cutoff of $\mathrm{P}<0.01$. The second condition was that the two genes that coded interacting proteins used to lay an edge between the two pathways had to be highly co-expressed, with an absolute value of Pearson's correlation coefficient $(\mathrm{IPCCl})>0.8$; if not, then the edge between two pathways would be rejected. Based on these requirements, a PIN was constructed. It is considered that if a network is too big that a considerable number of key genes and interactions are probably ignored (24). As a result, to reduce the complicated network, the scores of a pair of pathways in the PIN were calculated; defined as the summation of the absolute values of PCC for the PPIs in every two pathways. The top 5\% pathway interactions were chosen to construct an informative PIN for the detection of dysregulated pathways.

PCA analysis and selection of seed pathway. PCA is a dimension reduction approach that has been widely used in gene expression analysis (17); it is able to efficiently depict the internal structure of high-dimension data by preserving the variance within the data while converting the data into low-dimension space (25). In the present study, PCA was used to calculate pathway activity according to gene expression data from each pathway. Notably, all the genes were mapped to background pathways, and only those genes that aligned to the background pathways were kept for PCA analysis. The activity of each pathway (that is, the summary of the expression values of all genes in the given pathway) was calculated using the PCA method. The activity score for the corresponding pathway was determined as the first principal component from PCA. The activity score for a pathway between the experimental and the control groups was different, and the difference may demonstrate its correlation to the disease; that is, the greater the difference was, the more relevant this pathway was to the disease. Therefore, in the present study, the pathway with the maximum change of activity score between PMOP and control groups was selected as the seed pathway. 
Detecting dysregulated pathways from the informative PIN. Support vector machines (SVMs) were used to extract the dysregulated pathways. Briefly, an individual pathway that best distinguished disease from normal state was identified as the first pathway biosignature (hereafter called the seed pathway), and the second pathway that was able to integrate with the seed pathway to obtain better classification accuracy was extracted from the pathways that interacted with the seed pathway in the PIN. The process was repeated, and new pathways were added to the obtained pathway set until no more pathways could be assembled into the pathway set to enhance classification results. Classification accuracy was measured using the AUC index, based on five-fold cross validation. In an attempt to obtain robust biomarkers, five-fold cross-validation was repeated 100 times. The mean value was collected as the final result.

\section{Results}

Informative PIN construction. Based on the cutoff criteria of $\mathrm{P}<0.01$, a total of 544 DEGs between the PMOP group and the normal group were identified. The top 20 DEGs, in ascending order of P-values, are provided in Table I. The identified DEGs were used to select the interactions for establishing the PIN, as only the interactions in the background PPI data were able to meet at least one of the two conditions and were kept to build the PIN.

Based on the pre-defined threshold, a PIN that was involved in 54,517 pathway-pathway interactions was constructed. As very large networks may easily neglect a few significant interactions (23) they should be reduced in size. In the present study, the interactions with low $\mid \mathrm{PCCl}$ scores were removed, and the top $5 \%$ of all interactions were adopted for further analysis. A network comprising the top 5\% of interactions was defined as the informative PIN (Fig. 1). The informative PIN included 2,725 interactions between 1,189 pathways and was used to identify dysregulated pathways; specific information about the 1,189 pathways is not provided here. Based on the informative PIN, the strengths among the pathways were distinguishable. The weight of a pathway-pathway interaction was defined as the summation of $\mathrm{IPCCl}$ scores of all genes, and interactions having greater weight scores may be more important for PMOP compared with other interactions. The weight scores of the 2,725 interactions ranged between 95 and 323 . Notably, in the informative PIN, there were only 11 pairs of pathway interactions with weight scores $>300$.

Selection of the seed pathway. As there were differences between pathways in the informative PIN, how to assess the significance of each pathway and extract the significant pathway in the PIN was a challenge. The activity score for the 1,189 pathways was calculated using the PCA method to evaluate the significance. It was revealed that the pathways had different changes to their activity score between PMOP and normal groups (Fig. 2). The pathway with the maximum change in activity score between PMOP and normal groups was regarded as the seed pathway, which, in the present study, was identified as 'mitotic prometaphase'.

Detection of dysregulated pathways for PMOP. Starting with the seed pathway, a pathway set was identified by adding
Table I. List of the top 20 differentially expressed genes.

\begin{tabular}{ll}
\hline Gene & P-value \\
\hline KTN1 & $1.51 \times 10^{-05}$ \\
DKK1 & $1.62 \times 10^{-05}$ \\
ARHGAP42 & $1.93 \times 10^{-05}$ \\
ACSL3 & $2.23 \times 10^{-05}$ \\
GOLGA4 & $3.43 \times 10^{-05}$ \\
IFITM2 & $3.94 \times 10^{-05}$ \\
NNT-AS1 & $6.45 \times 10^{-05}$ \\
ABRA & $7.67 \times 10^{-05}$ \\
PRKAA2 & $9.22 \times 10^{-05}$ \\
DLEU2 & $1.10 \times 10^{-04}$ \\
SOST & $1.15 \times 10^{-04}$ \\
PDZRN3 & $1.39 \times 10^{-04}$ \\
KALRN & $1.56 \times 10^{-04}$ \\
FSTL3 & $1.68 \times 10^{-04}$ \\
WWP1 & $1.70 \times 10^{-04}$ \\
MUC17 & $2.17 \times 10^{-04}$ \\
ZAK & $2.41 \times 10^{-04}$ \\
ITM2A & $2.79 \times 10^{-04}$ \\
IPO7 & $2.87 \times 10^{-04}$ \\
ZNF787 & $2.99 \times 10^{-04}$ \\
\hline
\end{tabular}

pathways to increase the classification accuracy, and this procedure continued until the AUC index decreased. Using 'mitotic prometaphase' as the seed, a pathway set was identified that was involved in 8 dysregulated pathways with and AUC score of 0.85 . This pathway set included 'mitotic prometaphase', 'resolution of sister chromatid cohesion', 'transport of mature mRNA derived from an intron-containing transcript', 'interactions of Vpr with host cellular proteins', 'metabolism of non-coding RNA', 'regulation of HSF1-mediated heat shock response', 'mRNA splicing' and 'mRNA splicing-major pathway'. The effective classification ability of this method indicated that the detected dysregulated pathways may be utilized as robust signatures. A network of these eight dysregulated pathways is provided in Fig. 3 .

In addition, the genes enriched in the detected dysregulated pathways were compared with the identified DEGs (Table II). It was observed that only a small fraction, between 8.4 and $21.2 \%$, of the genes in the extracted dysregulated pathways overlapped with the DEGs. This phenomenon further demonstrated that a pathway as an entity may be better than a single gene at diagnosing complicated diseases, even if the genes enriched in the pathway were not differentially expressed.

\section{Discussion}

A number of previous studies suggested that pathway-based analyses may provide more reproducible results, relative to individual gene-based methods (26-28). For example, one previous study used pathways to compare different brain regions of patients with Alzheimer's disease and observed dysregulated pathways that cooperated in the different brain regions (29). However, the statistical significance of the pathway 


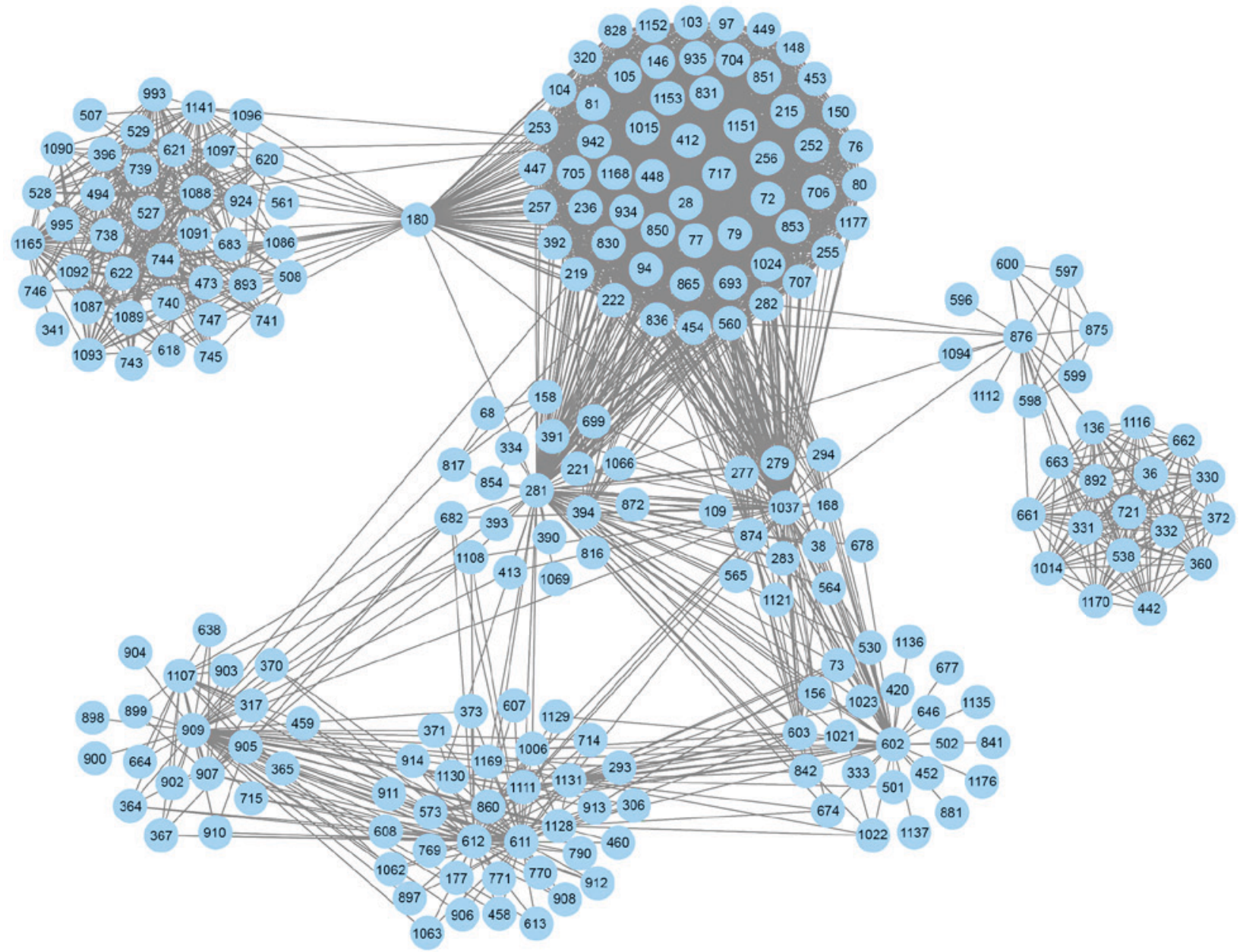

Figure 1. Pathway-interaction network for postmenopausal osteoporosis. Numbered nodes (blue circles) represent pathways and edges represent an interaction between two pathways.

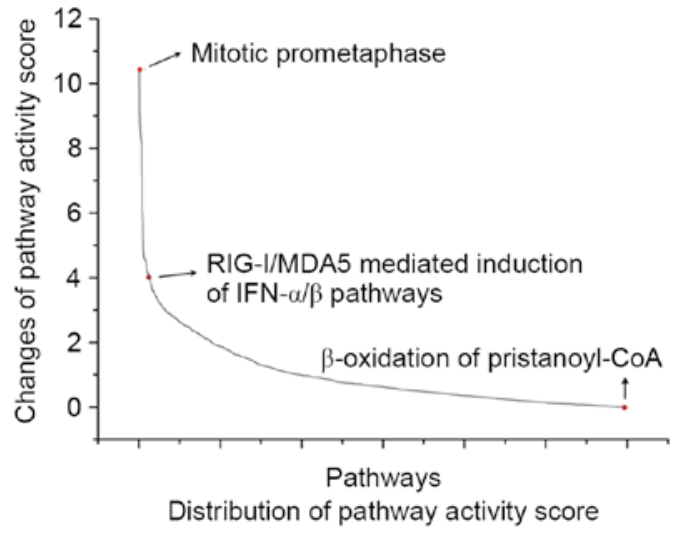

Figure 2. Distribution of the activity score changes of the 1,189 background pathways between postmenopausal osteoporosis and normal groups. Among these, the mitotic prometaphase pathway exhibited the most significant change of activity score between two groups was selected and called the seed pathway. CoA, coenzyme A; IFN, interferon; MDA-5, melanoma differentiation-associated 5; RIG-1, retinoic acid-inducible gene 1.

was evaluated using hypergeometric distribution and pathways were analyzed individually; the crosstalk among pathways was not considered. Notably, the identification of pathway crosstalk in specific conditions may benefit studies on pathway functions and the molecular mechanisms of biological processes (30).

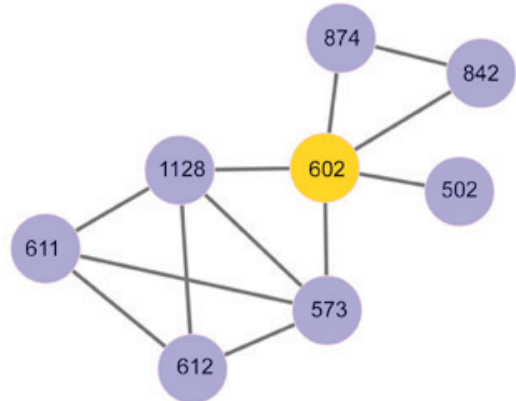

Figure 3. Dysregulated pathway interaction network in postmenopausal osteoporosis that is involved in eight dysregulated pathways, which were assembled into a pathway network based on these interactions. Each node represents a pathway; the yellow node represents the seed pathway (mitotic prometaphase), purple nodes represent the dysregulated pathways that interacted with the seed; numbers indicate the pathway ID (see Table II). The pathway ID was defined by the present study in alphabetical order.

Collectively, PPI data may provide valuable information regarding the interactions among functionalities that are vital to cell survival and growth. Network biology provides new opportunities to analyze the interaction data and gain insights into the mechanisms by which biological systems operate (31). For example, the characteristic sub-pathway network method was used to identify disease-specific pathway crosstalk by 
Table II. Dysregulated pathways extracted from the informative pathway-interaction network, and the DEGs involved in the dysregulated pathways.

\begin{tabular}{|c|c|c|c|c|}
\hline Index & Pathway & Genes (n) & DEGs (n) & DEGs \\
\hline 602 & Mitotic prometaphase & 105 & 11 & $\begin{array}{l}\text { PPP1CC, CLIP1, PLK1, CDC20, PPP2R5E, RCC2, } \\
\text { SKA2, PPP2CA, INCENP, CDCA } 8 \text { and NUF2 }\end{array}$ \\
\hline 874 & $\begin{array}{l}\text { Resolution of sister chromatid } \\
\text { cohesion }\end{array}$ & 97 & 11 & $\begin{array}{l}\text { PPP1CC, CLIP1, PLK1, CDC20, PPP2R5E, NUF2, } \\
\text { RCC2, SKA2, PPP2CA, INCENP and CDCA8 }\end{array}$ \\
\hline 1,128 & $\begin{array}{l}\text { Transport of mature mRNA derived } \\
\text { from an intron-containing transcript }\end{array}$ & 47 & 6 & SRSF11, NUP62, SRSF6, NUP54,AAAS and ALYREF \\
\hline 502 & $\begin{array}{l}\text { Interactions of } \mathrm{Vpr} \text { with host } \\
\text { cellular proteins }\end{array}$ & 33 & 7 & $\begin{array}{l}\text { NUP62, SLC25A4, HMGA1, BANF1, SLC25A6, } \\
\text { NUP54 and AAAS }\end{array}$ \\
\hline 573 & Metabolism of non-coding RNA & 47 & 6 & NUP62, SNRPD3, SNRPF, NUP54, AAAS and PHAX \\
\hline 842 & $\begin{array}{l}\text { Regulation of HSF1-mediated heat } \\
\text { shock response }\end{array}$ & 74 & 11 & $\begin{array}{l}\text { HSPA6, DNAJB1, RPA2, NUP62, HSPA2, BAG3, } \\
\text { AAAS, SIRT1, RPS19BP1, DNAJC2 and NUP54 }\end{array}$ \\
\hline 611 & mRNA splicing & 107 & 9 & $\begin{array}{l}\text { SRSF11, GTF2F1, SNRPD3, POLR2A, SNRPF, } \\
\text { ALYREF, SRSF6, SF3B4 and LSM2 }\end{array}$ \\
\hline 612 & mRNA splicing-major pathway & 107 & 9 & $\begin{array}{l}\text { SRSF11, GTF2F1, SNRPD3, POLR2A, SNRPF, } \\
\text { ALYREF, SRSF6, SF3B4 and LSM2 }\end{array}$ \\
\hline
\end{tabular}

'Pathway IDs (indexes)' are numbered based on the alphabetical order of pathways. DEG, differentially expressed gene.

counting 'active PPIs' (32). From a systematic perspective, analysis of disease-related interaction networks may improve our understanding of the complexity of biological pathways and may aid in the identification of the molecular processes of disease progression. Therefore, the present study used PINs to identify dysregulated pathways based on the crosstalk among pathways by integrating protein interaction data and cellular pathways. An advantage of this method is that, in cases in which pathways have only marginally enriched P-values, there may still be a strong signal if the pathways collectively form a compact module in the PIN.

With the goal of extracting dysregulated pathways in PMOP, and to further exploring the molecular mechanisms of PMOP, the present study constructed a PIN comprising 2,725 interactions between 1,189 pathways, based on gene expression profiles, pathway data and PPI information, and subsequently identified dysregulated pathways in the PIN according to classification accuracy using SVMs. The activity score for each pathway was calculated using the PCA approach, and the mitotic prometaphase pathway was identified and defined as the seed pathway. The selected pathway set included eight pathways, and among these eight dysregulated pathways, two pathways were related to mitotic activities and two pathways were associated with mRNA splicing.

As previously reported, the pre-mRNA splicing factor, cell division cycle 5-like, regulates mitotic progression (33). In addition, mitotic dysregulation may result in chromosomal pathology, which leads to further dysregulation of genes involved in the aging process, such as in OP and arthritis (34). Therefore, the present study hypothesized that dysregulated mRNA splicing and mitosis may serve important roles in the development of PMOP.

In eukaryotic cells, sister chromatid cohesion is required for the proper transmission of the replicated genome from generation to generation, and is dependent on cohesin proteins (35). The pre-mRNA-processing factor 19 (Prp19) complex serves important roles in pre-mRNA splicing (36), and inhibition of Prp19 has been demonstrated to affect the splicing of pre-mRNAs that encode proteins that are essential to sister chromatid cohesion, and thus indirectly cause defects in cohesion $(37,38)$. Another study also reported that the Prp19 splicing complex was necessary for sister chromatid cohesion during mitosis and maintenance of genome stability (39); genomic instability is considered to be associated with the aging process (40). OP is a common age-related disease; that the identified dysregulated pathways 'mRNA splicing' and 'resolution of sister chromatid cohesion' exhibited a close interaction suggested that this type of correlation may serve important roles in the development and progression of PMOP.

In conclusion, the present study identified a dysregulated pathway set for PMOP, which may shed light on the molecular mechanism of PMOP, and may present candidate signatures for targeted therapy for PMOP. However, several limitations of the present study must be taken into consideration. First, the sample size was relatively small. Second, how these pathways synergistically regulate PMOP progression at the molecular level remains unclear, and further investigations are required. Finally, analysis was based on existing data and only used a bioinformatics approach, with no experimental verification. Therefore, additional experimental investigations using animal or patient tissues are warranted to discover the potential alterations of these pathways and to further our understanding of the pathogenic mechanisms of PMOP.

\section{References}

1. Manolagas SC: Birth and death of bone cells: Basic regulatory mechanisms and implications for the pathogenesis and treatment of osteoporosis. Endocr Rev 21: 115-137, 2000.

2. Riggs BL: Involutional osteoporosis. N Engl J Med 314: 1676-1686, 1986. 
3. Seeman E: Bone quality: The material and structural basis of bone strength. J Bone Miner Metab 26: 1-8, 2008.

4. Marcus R: Post-menopausal osteoporosis. Best Pract Res Clin Obstet Gynaecol 16: 309-327, 2002.

5. Seibel MJ, Dunstan CR, Zhou H, Allan CM and Handelsman DJ: Sex steroids, not FSH, influence bone mass. Cell 127: 1079-1081, 2006.

6. Hernlund E, Svedbom A, Ivergård M, Compston J, Cooper C, Stenmark J, McCloskey EV, Jönsson B and Kanis JA: Osteoporosis in the European Union: Medical management, epidemiology and economic burden. A report prepared in collaboration with the International Osteoporosis Foundation (IOF) and the European Federation of Pharmaceutical Industry Associations (EFPIA) Arch Osteoporos 8: 136, 2013.

7. Burge R, Dawson-Hughes B, Solomon DH, Wong JB, King A and Tosteson A: Incidence and economic burden of osteoporosis-related fractures in the United States, 2005-2025. J Bone Miner Res 22: 465-475, 2007.

8. Michaëlsson K, Melhus H, Ferm H, Ahlbom A and Pedersen NL: Genetic liability to fractures in the elderly. Arch Intern Med 165 1825-1830, 2005

9. Guo Y, Dong SS, Chen XF, Jing YA, Yang M, Yan H, Shen H, Chen XD, Tan LJ, Tian Q, et al: Integrating epigenomic elements and GWASs identifies BDNF gene affecting bone mineral density and osteoporotic fracture risk. Sci Rep 6: 30558, 2016.

10. Wang C, Zhang Z, Zhang H, He JW, Gu JM, Hu WW, Hu YQ, Li M, Liu YJ, Fu WZ, et al: Susceptibility genes for osteoporotic fracture in postmenopausal Chinese women. J Bone Miner Res 27: 2582-2591, 2012

11. Geng L, Yao Z, Yang H, Luo J, Han L and Lu Q: Association of CA repeat polymorphism in estrogen receptor $\beta$ gene with postmenopausal osteoporosis in Chinese. J Genet Genomics 34: 868-876, 2007

12. Song JF, Jing ZZ, Hu W and Su YX: Association between single nucleotide polymorphisms of the osteoprotegerin gene and postmenopausal osteoporosis in Chinese women. Genet Mol Res 12: 3279-3285, 2013.

13. Xu XJ, Shen L, Yang YP, Zhu R, Shuai B, Li CG and $\mathrm{Wu}$ MX: Serum $\beta$-catenin levels associated with the ratio of RANKL/OPG in patients with postmenopausal osteoporosis. Int J Endocrinol 2013: 534352, 2013.

14. Feng J, Liu S, Ma S, Zhao J, Zhang W, Qi W, Cao P, Wang Z and Lei W: Protective effects of resveratrol on postmenopausal osteoporosis: Regulation of SIRT1-NF- $\kappa$ B signaling pathway. Acta Biochim Biophys Sin (Shanghai) 46: 1024-1033, 2014.

15. Reppe S, Sachse D, Olstad OK, Gautvik VT, Sanderson P, Datta HK, Berg JP and Gautvik KM: Identification of transcriptional macromolecular associations in human bone using browser based in silico analysis in a giant correlation matrix. Bone 53 : 69-78, 2013.

16. Liu Y, Wang Y, Yang N, Wu S, Lv Y and Xu L: In silico analysis of the molecular mechanism of postmenopausal osteoporosis Mol Med Rep 12: 6584-6590, 2015.

17. Hotelling $\mathrm{H}$ : Analysis of a complex of statistical variables into principal components. J Edu Psychol 24: 417, 1933.

18. Gautier L, Cope L, Bolstad BM and Irizarry RA: Affy-analysis of Affymetrix GeneChip data at the probe level. Bioinformatics 20 307-315, 2004.

19. Irizarry RA, Hobbs B, Collin F, Beazer-Barclay YD, Antonellis KJ, Scherf U and Speed TP: Exploration, normalization, and summaries of high density oligonucleotide array probe level data. Biostatistics 4: 249-264, 2003.

20. Bolstad BM, Irizarry RA, Astrand M and Speed TP: A comparison of normalization methods for high density oligonucleotide array data based on variance and bias. Bioinformatics 19: 185-193, 2003.
21. Joshi-Tope G, Gillespie M, Vastrik I, D'Eustachio P, Schmidt E, de Bono B, Jassal B, Gopinath GR, Wu GR, Matthews L, et al: Reactome: A knowledgebase of biological pathways. Nucleic Acids Res 33 (Database Issue): D428-D432, 2005.

22. Ahn T, Lee E, Huh N and Park T: Personalized identification of altered pathways in cancer using accumulated normal tissue data. Bioinformatics 30: i422-i429, 2014

23. Franceschini A, Szklarczyk D, Frankild S, Kuhn M, Simonovic M, Roth A, Lin J, Minguez P, Bork P, von Mering C and Jensen LJ: STRING v9.1: Protein-protein interaction networks, with increased coverage and integration. Nucleic Acids Res 41 (Database Issue): D808-D815, 2013.

24. Nibbe RK, Chowdhury SA, Koyutürk M, Ewing R and Chance MR: Protein-protein interaction networks and subnetworks in the biology of disease. Wiley Interdiscip Rev Syst Biol Med 3: 357-367, 2011.

25. Sharov AA, Dudekula DB and Ko MS: A web-based tool for principal component and significance analysis of microarray data. Bioinformatics 21: 2548-2549, 2005

26. Segal E, Friedman N, Kaminski N, Regev A and Koller D: From signatures to models: Understanding cancer using microarrays. Nat Genet 37 (Suppl): S38-S45, 2005.

27. Wang X, Ding M, Yang Y, Feng Y, Shi Z, Qiu F and Zhu M: Personalized discovery of altered pathways in clear cell renal cell carcinoma using accumulated normal sample data. J BUON 21: 390-398, 2016

28. Glazko GV and Emmert-Streib F: Unite and conquer: Univariate and multivariate approaches for finding differentially expressed gene sets. Bioinformatics 25: 2348-2354, 2009.

29. Liu ZP, Wang Y, Zhang XS and Chen L: Identifying dysfunctional crosstalk of pathways in various regions of Alzheimer's disease brains. BMC Syst Biol 4 (Suppl 2): S11, 2010.

30. Li Y and Agarwal P: A pathway-based view of human diseases and disease relationships. PLoS One 4: e4346, 2009.

31. Xia Y, Yu H, Jansen R, Seringhaus M, Baxter S, Greenbaum D, Zhao $\mathrm{H}$ and Gerstein M: Analyzing cellular biochemistry in terms of molecular networks. Biochemistry 73: 1051-1087, 2004.

32. Huang Y and Li S: Detection of characteristic sub pathway network for angiogenesis based on the comprehensive pathway network. BMC Bioinformatics 11 (Suppl 1): S32, 2010.

33. Mu R, Wang YB, Wu M, Yang Y, Song W, Li T, Zhang WN, Tan B, Li AL, Wang N, et al: Depletion of pre-mRNA splicing factor $\mathrm{Cdc} 5 \mathrm{~L}$ inhibits mitotic progression and triggers mitotic catastrophe. Cell Death Dis 5: e1151, 2014.

34. Ly DH, Lockhart DJ, Lerner RA and Schultz PG: Mitotic misregulation and human aging. Science 287: 2486-2492, 2000.

35. Losada A, Hirano M and Hirano T: Identification of Xenopus SMC protein complexes required for sister chromatid cohesion. Genes Dev 12: 1986-1997, 1998.

36. Tarn WY, Lee KR and Cheng SC: The yeast PRP19 protein is not tightly associated with small nuclear RNAs, but appears to associate with the spliceosome after binding of U2 to the pre-mRNA and prior to formation of the functional spliceosome. Mol Cell Biol 13: 1883-1891, 1993.

37. Neumann B, Walter T, Hériché JK, Bulkescher J, Erfle H, Conrad C, Rogers P, Poser I, Held M, Liebel U, et al: Phenotypic profiling of the human genome by time-lapse microscopy reveals cell division genes. Nature 464: 721-727, 2010.

38. Hofmann JC, Tegha-Dunghu J, Dräger S, Will CL, Lührmann R and Gruss OJ: The Prp19 complex directly functions in mitotic spindle assembly. PLoS One 8: e74851, 2013.

39. Watrin E, Demidova M, Watrin T, Hu Z and Prigent C: Sororin pre-mRNA splicing is required for proper sister chromatid cohesion in human cells. EMBO Rep 15: 948-955, 2014.

40. Vijg J and Suh Y: Genome instability and aging. Annu Rev Physiol 75: 645-668, 2013. 